\title{
Distributed circuits underlying anxiety
}

\author{
Avishek Adhikari* \\ Deisseroth Laboratory, CNC Program, Bioengineering Department, Stanford University, Palo Alto, CA, USA
}

Edited by:

Mazen A. Kheirbek, Columbia

University/Research Foundation for

Mental Hygiene, USA

Reviewed by:

Gregg Stanwood, Vanderbilt

University, USA

Nesha Burghardt, Research

Foundation for Mental Hygiene, USA

${ }^{*}$ Correspondence:

Avishek Adhikari, Deisseroth

Laboratory, CNC Program,

Bioengineering Department, Stanford

University, 1050 Arastradero Road,

BLDG B, Room 233, Palo Alto, CA

94306, USA

e-mail: avishek@stanford.edu
Anxiety is of paramount importance for animals, as it allows assessment of the environment while minimizing exposure to potential threats. Furthermore, anxiety disorders are highly prevalent. Consequently, the neural circuitry underlying anxiety has been a topic of great interest. In this mini review, we will discuss current views on anxiety circuits. We will focus on rodent anxiety paradigms, but we will also consider results from human neuroimaging and clinical studies. We briefly review studies demonstrating the central role that the amygdala and the bed nucleus of the stria terminals (BNST) play in modulating anxiety and present evidence showing how the bed nucleus uses different output pathways to influence specific features of anxiolysis. Lastly, we propose that several brain regions, such as the medial prefrontal cortex (mPFC) and the ventral hippocampus (VHPC), act in a coordinated fashion with the amygdala and BNST, forming a distributed network of interconnected structures that control anxiety both in rodents and humans.

Keywords: anxiety, amygdala, BNST, ventral hippocampus, medial prefrontal cortex

\section{INTRODUCTION}

In the clinical literature anxiety is defined as a long-term trait characterized by non-adaptive hypervigilance and overestimation of the potential for threat in uncertain situations (Sylvers et al., 2011). On the other hand, in the animal literature anxiety is often defined as a temporary behavioral state induced by diffuse threatening stimuli (Sylvers et al., 2011), such as open spaces (Pellow et al., 1985) and bright lights (Crawley, 1985). Despite these differences, similar brain structures underlie both human (Yassa et al., 2012; Boehme et al., 2013) and rodent (Moreira et al., 2007; Duvarci et al., 2009) measures of anxiety, and drugs that are anxiolytic in humans decrease avoidance towards open spaces and bright lights in rodents (File and Pellow, 1985; Walker and Davis, 1997a; Schmitt and Hiemke, 1998). These similarities strongly suggest that the study of rodent anxiety paradigms can provide insights into human anxiety disorders.

Considering anxiety disorders generate large financial and emotional burdens (Lecrubier, 2007), it is unsurprising that the circuits underlying anxiety have received tremendous attention. Here, we will cover a few topics relating to anxiety. We will first introduce common rodent anxiety paradigms and discuss the role of the amygdala and the bed nucleus of the stria terminalis (BNST) in anxiety. Then, we will discuss evidence showing that the BNST and the amygdala are part of a larger network that modulates anxiety, involving the ventral hippocampus (vHPC), the medial prefrontal cortex (mPFC) and other regions.

\section{ANIMAL MODELS OF ANXIETY}

In humans anxiety is assessed by self-report. While this method cannot be used in rodents, one can measure their innate avoidance of bright lights and open spaces. Rodents avoid open areas and brightness presumably because they are more vulnerable to predators. Avoidance towards openness is commonly studied in the elevated plus maze, a paradigm consisting of a plus-shaped maze on an elevated platform. It contains two open arms without walls and two closed arms which are enclosed by high walls. Rodents innately avoid the open arms (Pellow et al., 1985). Another similar paradigm is the open field, which consists of an enclosure with high walls. As expected, rodents spend more time exploring the walled periphery of the open field and avoid its exposed center. This avoidance behavior has pharmacological validity, as drugs that decrease anxiety in humans, such as benzodiazepines, decrease the aversion rodents have towards open spaces (File and Pellow, 1985; Schmitt and Hiemke, 1998).

Rodents also show innate aversion to bright lights. This behavior has been studied using the startle paradigm, in which rodents display a startle reflex when presented with an unpredictable burst of noise. Interestingly, the amplitude of the startle is enhanced after rats are presented with an innately aversive stimulus, such as a cat (Blundell et al., 2005) or bright illumination (Walker and Davis, 1997a). This paradigm has been validated pharmacologically, as the amplitude of the light-potentiated startle is decreased by the anxiolytic drug buspirone (Walker and Davis, 1997a). While there are other paradigms that measure different types of anxiety, such as social anxiety (Pobbe et al., 2011), here, we will focus on paradigms measuring aversion towards open spaces and bright lights as these are among the most common anxiety paradigms.

\section{THE AMYGDALA IN ANXIETY}

A wealth of data implicates the amygdala in anxiety both in humans and rodents. For example, higher amygdala volume is correlated with more anxiety in humans (Qin et al., 2013; Machado-de-Sousa et al., 2014), and patients with social anxiety 
disorder show increased amygdala activation during anticipatory anxiety relative to healthy subjects (Boehme et al., 2013). Moreover, immediate early gene assays in rodents show amygdala activation following exposure to anxiogenic contexts (Silveira et al., 1993; Butler et al., 2012), and pharmacological inactivation of the amygdala is anxiolytic in the elevated plus maze (Moreira et al., 2007). Thus, both human and rodent studies show that the amygdala is a crucial node in the anxiety circuitry.

The most studied amygdala sub-regions are the basolateral amygdala (BLA) and the central nucleus of the amygdala. Results from the fear conditioning literature show the BLA integrates highly processed information about the environment and encodes behaviorally relevant cues (LeDoux, 2000). Indeed, the BLA has cells that respond to cues that predict threats (Amano et al., 2011) and cells that respond to cues that no longer predict danger (Senn et al., 2014). The BLA robustly projects to the central nucleus of the amygdala (Pitkanen, 2000; Tye et al., 2011) and its main excitatory input (LeDoux, 2000). The central nucleus of the amygdala is comprised of central lateral and central medial portions. The central lateral amygdala inhibits the central medial nucleus (Jolkkonen and Pitkänen, 1998; Tye et al., 2011), which is the main output of the amygdala (LeDoux, 2000). A crucial role for the central nucleus of the amygdala in mediating behaviors induced by threatening stimuli was demonstrated by a study showing decreased freezing to a tone that predicts a shock following central medial nucleus inactivation (Ciocchi et al., 2010). This effect is presumably mediated by projections of the central medial nucleus to hypothalamic and brain stem targets (Price and Amaral, 1981) which modulate various features of the anxious state.

Local pharmacological inactivation and lesion studies indicate that the central nucleus of the amygdala, but not the BLA, is required for avoidance of open spaces (Moller et al., 1997; Moreira et al., 2007; Carvalho et al., 2012). However, these results may reflect compensatory changes after drug infusions or lesions. Fortunately, optogenetics allows researchers to overcome these limitations as optogenetic manipulations are quick and reversible, and are thus less likely to be confounded by slow compensatory changes. It was shown that optogenetic activation of the entire BLA augments anxiety, while selective activation of the projection from the BLA to the central lateral nucleus decreases anxiety (Tye et al., 2011). These results make anatomical sense, as the central lateral nucleus inhibits the central medial nucleus (Tye et al., 2011), which is the main output structure of the amygdala (Price and Amaral, 1981). These data indicate that classifying an entire amygdala region as anxiogenic or anxiolytic is an oversimplification, as different cells in the same region can have different functions depending on their post-synaptic targets (Tye et al., 2011).

It is noteworthy that the amygdala has other regions besides the BLA and the central nucleus of the amygdala, which also mediate defensive behaviors. For example, the medial amygdala is required to react to olfactory cues from a predator (Li et al., 2004), whereas the basomedial amygdala mediates avoidance of potentially threatening auditory and visual cues (Gross and Canteras, 2012).

In summary, prior reports demonstrate the amygdala is crucial for generating anxiety. However, some sub-regions have been more extensively studied than others. Furthermore, the study of functional differentiation among subpopulations of cells in the same amygdala region has only just begun.

\section{BEYOND THE AMYGDALA: THE EXTENDED AMYGDALA}

The prominent role of the amygdala in mediating anxiety motivated researchers to identify other amygdala-associated structures that also influence anxiety. Anatomical studies suggest the BNST modulates anxiety, as it receives prominent projections from the amygdala (De Olmos, 1972; Dong et al., 2001a) and projects to many hypothalamic and brainstem structures (De Olmos and Ingram, 1972; Holstege et al., 1985; Dong et al., 2001b; Dong and Swanson, 2004) that receive central amygdala (CeA) nucleus terminals (De Olmos, 1972; LeDoux, 2000). Furthermore, the central nucleus of the amygdala and the BNST are also similar in their neuropeptide expression profile (Roberts et al., 1982; Woodhams et al., 1983) and morphology (McDonald, 1983). Noting these similarities, Alheid et al proposed that the BNST is part of the extended amygdala, a group of anatomically and functionally related structures which include the BNST and the central nucleus of the amygdala (Alheid and Heimer, 1988; Alheid et al., 1998).

Like the amygdala, a large body of evidence implicates the BNST in anxiety. For example, compared to control subjects, patients with generalized anxiety disorder show hyperactivation of the BNST when participating in a gambling task with high uncertainty (Yassa et al., 2012). Moreover, the BNST is recruited during hypervigilance in individuals with higher traitanxiety (Somerville et al., 2010). Thus, human imaging studies suggest that BNST activity is correlated with increased anxiety. The rodent literature, however, presents conflicting results. For example, Treit et al. (1998) found no effect of BNST lesions in open-arm avoidance in the elevated plus maze, whereas Duvarci et al. (2009) reported anxiogenic effects of BNST lesions on the plus maze. Furthermore, van Dijk et al. found that electrical stimulation of the BNST did not alter behavior in the plus-maze (van Dijk et al., 2013), and Walker reported that infusions of glutamate antagonists in the BNST are anxiolytic in the light potentiated startle paradigm (Walker and Davis, 1997b). These data show the BNST modulates anxiety in rodents, but they do not make it clear if this structure increases or decreases anxiety.

The anatomy of the BNST provides a potential explanation for these discrepancies. Anatomists have recognized that the BNST is composed of several sub-nuclei differing in anatomical (Dong et al., 2001b; Dong and Swanson, 2004) and neurochemical features (Walter et al., 1991), which likely reflect functional differentiation among BNST nuclei. If different BNST subregions regulate anxiety in opposite directions, lesion and pharmacology studies affecting distinct subregions of the BNST could provide conflicting results. Supporting this view, Kim et al. showed that optogenetic inactivation of the oval nucleus of the BNST is anxiolytic, while decreases in activity in the anterodorsal BNST (adBNST) are anxiogenic (Kim et al., 2013). These results demonstrate that different subregions of the BNST have different functions, and suggest an explanation for the discrepancies found between previous studies (Treit et al., 1998; Duvarci et al., 2009). 
BNST cells may also differ in their neurochemical profiles (Walter et al., 1991), suggesting functional differentiation among cells located in the same BNST nucleus. Indeed, Jennings et al. (2013b) showed that the firing rate of BNST glutamatergic neurons increased when mice were presented with aversive stimuli, whereas BNST GABAergic neurons decreased firing in the same condition. Furthermore, optogenetic activation of the projection from glutamatergic BNST cells to the ventral tegmental area (VTA) was anxiogenic and aversive, while activation of the projection of GABAergic BNST cells to the VTA was anxiolytic and rewarding (Jennings et al., 2013b). A role for the BNST in reward is also suggested by its involvement in stress-induced reinstatement of cocaine seeking (Erb and Stewart, 1999). This finding may partially explain the correlation between trait anxiety and severity of drug addiction (O'Leary et al., 2000). Lastly, it has also been shown that activation of axon terminals of GABAergic BNST neurons in the lateral hypothalamus robustly increases eating in mice (Jennings et al., 2013a). This effect may play a role in the weight and feeding abnormalities seen in patients with anxiety disorders (Deboer and Smits, 2013). These results show that the neurochemical identity of BNST neurons is reflected in important functional differences. It is noteworthy that various neuropeptides, such as enkephalin (Arluison et al., 1990), neuropeptide Y and substance P (Walter et al., 1991) are richly expressed in this structure. Future studies are needed to identify the role of these neurotransmitters in anxiety.

Taken together, these data show that subregion specificity, neurochemical composition and anatomical connectivity are all features to be considered when studying the BNST. Indeed, optogenetic manipulation of specific anatomical projections, such as activation of BNST projections to the VTA (Jennings et al., 2013b) and of BLA terminals in the BNST (Kim et al., 2013) modulated anxiety, suggesting that a network of several brain regions acts together to dynamically fine-tune the expression of different features of anxiety according to ever-changing environmental demands.

\section{A DISTRIBUTED NETWORK UNDERLYING ANXIETY}

The view that a distributed network of interconnected brain regions modulates anxiety is not new and has been suggested previously (Papez, 1995). Here, we will briefly review recent evidence supporting this view, focusing on rodent in vivo electrophysiology papers, projection-targeting optogenetic studies, and neuroimaging reports.

Rodent in vivo recordings suggest the interplay between the mPFC, the BLA, and the vHPC affects anxiety. The first in vivo recording supporting this idea came from a fear conditioning study showing that BLA-hippocampus synchrony increases while animals freeze to a shock-predicting auditory tone (Seidenbecher et al., 2003). More recently, it has been shown that mPFC neurons encode arm type in the plus maze, by being preferentially active in either the closed or open arms, and the neurons that encode arm type most strongly also are more synchronized to vHPC activity (Adhikari et al., 2011). This result suggests the vHPC-mPFC pathway encodes aspects of the context relevant to anxiety. Furthermore, neural synchrony between the vHPC and the $\mathrm{mPFC}$ in the $\theta$-range $(4-12 \mathrm{~Hz})$ increases while mice explore the elevated plus maze or the open field compared to a familiar environment (Adhikari et al., 2010). Additionally, in the open field the amount of increase in mPFC-BLA $\theta$-range synchrony correlates with higher avoidance of the center of the open field (Likhtik et al., 2014). Lastly, mPFC-BLA and vHPC-mPFC synchrony increase before the animal transitions from dangerous to safe zones in anxiety paradigms (Adhikari et al., 2010; Likhtik et al., 2014), suggesting synchrony between these regions could influence exploration in an anxiogenic environment. In summary, rodent electrophysiology studies indicate a network of structures modulates anxiety.

However, these data are correlative and cannot demonstrate whether interactions among these brain regions affect anxiety. Perturbative methods are needed to investigate this question. To this end, it has been shown that local injections of gap junction blockers in the vHPC reduced both vHPC-mPFC synchrony and anxiety in the elevated plus maze and open field (Schoenfeld et al., 2013). The role of the BLA-vHPC pathway has also been studied through perturbative methods. It has been reported that optogenetic activation of the BLA-vHPC projection increases anxiety, whereas inhibition of this projection was anxiolytic in the elevate plus maze and open field (Felix-Ortiz et al., 2013). Just as the BLA, the BNST also has a prominent role in the anxiety network. Optogenetic activation of the adBNST-lateral hypothalamus projection was anxiolytic in the plus maze and open field, whereas activation of the adBNST-parabrachial nucleus (PB) projection selectively decreases respiration rate (a physiological marker of anxiety), but not behavior. Lastly, optogenetic activation of the adBNSTVTA pathway selectively induces conditioned place preference, as mice spend more time in a compartment in which the adBNTVTA projection was activated (Kim et al., 2013). These data show different outputs from the BNST control distinct features of anxiolysis. Thus, both rodent in vivo physiology and optogenetic studies suggest several interconnected regions influence anxiety.

Evidence from human neuroimaging studies also support this view. For example, co-activation of the amygdala and the insular cortex has been reported during negative affective states (Kober et al., 2008). This work also identified the periaqueductal gray, the hypothalamus and the amygdala as part of a limbic network in which members are co-activated, and which may be linked to the generation of negative valence. The authors also identify the hippocampus and the mPFC as part of a separate co-activated network, which can modulate and be modulated by the limbic network. Lastly, similar to results in rodents, activity in the anterior hippocampus (the human analog of the rodent vHPC) may have a role in anxiety, as its activity is correlated with trait anxiety (Satpute et al., 2012). These data suggest that the brain structures regulating anxiety are well-conserved between rodents and humans and indicate that a distributed network modulates anxiety (Figure 1). Although the precise role of each structure in the network is not known, one possibility is that contextual and sensory input from the mPFC and the vHPC is integrated by the BLA, which then drives the central nucleus of the amygdala and the BNST. These two structures, in turn, may activate downstream regions which control anxiety-related symptoms.

Importantly, prior studies have implicated other regions in anxiety, such as the medial septum. It has been shown that septal 


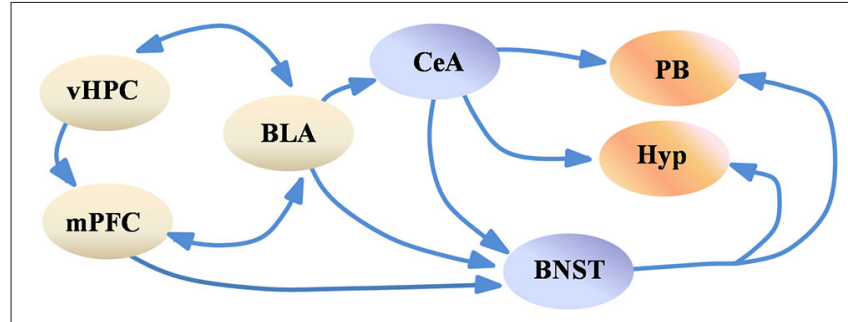

FIGURE 1 | Simplified scheme of the anxiety network. Highly processed sensory and contextual information from the VHPC and the MPFC is integrated by the BLA, which in turn may activate the CeA and the BNST. The CeA and the BNST project to the hypothalamus and to brain stem nuclei such as the PB, which modulate various features of anxiety, such as avoidance of open spaces and changes in respiration rate. For simplification purposes subregions of the BNST, CeA, Hypothalamus and mPFC are not shown. Abbreviations: BLA: basolateral amygdala, BNST: bed nucleus of the stria terminalis, CeA: central amygdala, Hyp: hypothalamus, mPFC: medial prefrontal cortex, PB: parabrachial nucleus, vHPC: ventral hippocampus.

inactivations or lesions decrease anxiety in the plus maze (Menard and Treit, 1996; Degroot and Treit, 2004). It is possible that the effect of medial septal lesions on anxiety is mediated by disruption of $\theta$-oscillations, as the medial septum controls hippocampal $\theta$ rhythms (Smythe et al., 1992). $\Theta$-activity is strongly implicated in anxiety, as $\theta$-range synchrony between the vHPC, mPFC and BLA is seen during high anxiety in rodents (Adhikari et al., 2010; Likhtik et al., 2014), and all clinically effective anxiolytics inhibit $\theta$-activity (McNaughton and Gray, 2000). Other regions implicated in anxiety include the insula (Kober et al., 2008), the nucleus basalis magnocellularis (Privou et al., 1998) and the ventral striatum (Kabli et al., 2013), among others. Future studies will identify all the components of this network and dissect the function of each projection. Lastly, one must consider that even though anxiety consists of multiple features (behavioral, hormonal, etc.), other equally complex constructs are influenced by anxiety through unknown mechanisms. These include aggression (Tang et al., 2013), risk-taking (de Visser et al., 2010) and feeding (Deboer and Smits, 2013). Investigating these outstanding questions may provide insights leading to novel therapies for anxiety disorders.

\section{AUTHOR CONTRIBUTIONS}

Avishek Adhikari wrote the manuscript.

\section{ACKNOWLEDGMENTS}

We thank members of the Deisseroth and Gordon labs for helpful discussions and K. Zalocusky and T. Stroh-Spijer for editing the manuscript. Avishek Adhikari is supported by a Walter V. and Idun Berry postdoctoral fellowship.

\section{REFERENCES}

Adhikari, A., Topiwala, M. A., and Gordon, J. A. (2010). Synchronized activity between the ventral hippocampus and the medial prefrontal cortex during anxiety. Neuron 65, 257-269. doi: 10.1016/j.neuron.2009.12.002

Adhikari, A., Topiwala, M. A., and Gordon, J. A. (2011). Single units in the medial prefrontal cortex with anxiety-related firing patterns are preferentially influenced by ventral hippocampal activity. Neuron 71, 898-910. doi: 10.1016/j. neuron.2011.07.027
Alheid, G. F., Beltramino, C. A., De Olmos, J. S., Forbes, M. S., Swanson, D. J., and Heimer, L. (1998). The neuronal organization of the supracapsular part of the stria terminalis in the rat: the dorsal component of the extended amygdala. Neuroscience 84, 967-996. doi: 10.1016/s0306-4522(97)00 $560-5$

Alheid, G. F., and Heimer, L. (1988). New perspectives in basal forebrain organization of special relevance for neuropsychiatric disorders: the striatopallidal, amygdaloid, and corticopetal components of substantia innominata. Neuroscience 27, 1-39. doi: 10.1016/0306-4522(88)90217-5

Amano, T., Duvarci, S., Popa, D., and Pare, D. (2011). The fear circuit revisited: contributions of the basal amygdala nuclei to conditioned fear. J. Neurosci. 31, 15481-15489. doi: 10.1523/JNEUROSCI.3410-11.2011

Arluison, M., Vankova, M., Cesselin, F., and Leviel, V. (1990). Origin of some enkephalin-containing afferents to the ventro-medial region of the globus pallidus in the rat. Brain Res. Bull. 25, 25-34. doi: 10.1016/0361-9230(90)90 $249-\mathrm{y}$

Blundell, J., Adamec, R., and Burton, P. (2005). Role of NMDA receptors in the syndrome of behavioral changes produced by predator stress. Physiol. Behav. 86, 233-243. doi: 10.1016/j.physbeh.2005.07.012

Boehme, S., Ritter, V., Tefikow, S., Stangier, U., Strauss, B., Miltner, W. H., et al. (2013). Brain activation during anticipatory anxiety in social anxiety disorder. Soc. Cogn. Affect. Neurosci. doi: 10.1093/scan/nst129. [Epub ahead of print].

Butler, R. K., White, L. C., Frederick-Duus, D., Kaigler, K. F., Fadel, J. R., and Wilson, M. A. (2012). Comparison of the activation of somatostatin- and neuropeptide Y-containing neuronal populations of the rat amygdala following two different anxiogenic stressors. Exp. Neurol. 238, 52-63. doi: 10.1016/j. expneurol.2012.08.002

Carvalho, M. C., Moreira, C. M., Zanoveli, J. M., and Brandao, M. L. (2012). Central, but not basolateral, amygdala involvement in the anxiolytic-like effects of midazolam in rats in the elevated plus maze. J. Psychopharmacol. 26, 543-554. doi: $10.1177 / 0269881110389209$

Ciocchi, S., Herry, C., Grenier, F., Wolff, S. B., Letzkus, J. J., Vlachos, I., et al. (2010). Encoding of conditioned fear in central amygdala inhibitory circuits. Nature 468, 277-282. doi: 10.1038/nature09559

Crawley, J. N. (1985). Exploratory behavior models of anxiety in mice. Neurosci. Biobehav. Rev. 9, 37-44. doi: 10.1016/0149-7634(85)90030-2

De Olmos, J. S. (1972). "The amygdaloid projection field in the rat as studied with the cupric-silver method," in The Neurobiology of the Amygdala, ed B. E. Eleftheriou (New York: Plenum Press), 145-204.

De Olmos, J. S., and Ingram, W. R. (1972). The projection field of the stria terminalis in the rat brain. An experimental study. J. Comp. Neurol. 146, $303-$ 334. doi: $10.1002 /$ cne. 901460303

de Visser, L., van der Knaap, L. J., van de Loo, A. J., van der Weerd, C. M., Ohl, F., and van den Bos, R. (2010). Trait anxiety affects decision-making differently in healthy men and women: towards gender-specific endophenotypes of anxiety. Neuropsychologia 48, 1598-1606. doi: 10.1016/j.neuropsychologia.2010. 01.027

Deboer, L. B., and Smits, J. A. (2013). Anxiety and disordered eating. Cognit. Ther. Res. 37, 887-889. doi: 10.1007/s10608-013-9565-9

Degroot, A., and Treit, D. (2004). Anxiety is functionally segregated within the septo-hippocampal system. Brain Res. 1001, 60-71. doi: 10.1016/j.brainres.2003. 10.065

Dong, H. W., Petrovich, G. D., and Swanson, L. W. (2001a). Topography of projections from amygdala to bed nuclei of the stria terminalis. Brain Res. Brain Res. Rev. 38, 192-246. doi: 10.1016/s0165-0173(01)00079-0

Dong, H. W., Petrovich, G. D., Watts, A. G., and Swanson, L. W. (2001b). Basic organization of projections from the oval and fusiform nuclei of the bed nuclei of the stria terminalis in adult rat brain. J. Comp. Neurol. 436, 430-455. doi: 10. 1002/cne.1079

Dong, H. W., and Swanson, L. W. (2004). Organization of axonal projections from the anterolateral area of the bed nuclei of the stria terminalis. J. Comp. Neurol. 468, 277-298. doi: 10.1002/cne.10949

Duvarci, S., Bauer, E. P., and Pare, D. (2009). The bed nucleus of the stria terminalis mediates inter-individual variations in anxiety and fear. J. Neurosci. 29, 1035710361. doi: 10.1523/JNEUROSCI.2119-09.2009

Erb, S., and Stewart, J. (1999). A role for the bed nucleus of the stria terminalis, but not the amygdala, in the effects of corticotropin-releasing factor on stressinduced reinstatement of cocaine seeking. J. Neurosci. 19:RC35. 
Felix-Ortiz, A. C., Beyeler, A., Seo, C., Leppla, C. A., Wildes, C. P., and Tye, K. M. (2013). BLA to vHPC inputs modulate anxiety-related behaviors. Neuron 79, 658-664. doi: 10.1016/j.neuron.2013.06.016

File, S. E., and Pellow, S. (1985). The effects of triazolobenzodiazepines in two animal tests of anxiety and in the holeboard. Br. J. Pharmacol. 86, 729-735. doi: 10.1111/j.1476-5381.1985.tb08952.x

Gross, C. T., and Canteras, N. S. (2012). The many paths to fear. Nat. Rev. Neurosci. 13, 651-658. doi: 10.1038/nrn3301

Holstege, G., Meiners, L., and Tan, K. (1985). Projections of the bed nucleus of the stria terminalis to the mesencephalon, pons, and medulla oblongata in the cat. Exp. Brain Res. 58, 379-391. doi: 10.1007/bf00235319

Jennings, J. H., Rizzi, G., Stamatakis, A. M., Ung, R. L., and Stuber, G. D. (2013a). The inhibitory circuit architecture of the lateral hypothalamus orchestrates feeding. Science 341, 1517-1521. doi: 10.1126/science.1241812

Jennings, J. H., Sparta, D. R., Stamatakis, A. M., Ung, R. L., Pleil, K. E., Kash, T. L., et al. (2013b). Distinct extended amygdala circuits for divergent motivational states. Nature 496, 224-228. doi: 10.1038/nature12041

Jolkkonen, E., and Pitkänen, A. (1998). Intrinsic connections of the rat amygdaloid complex: projections originating in the central nucleus. J. Comp. Neurol. 395, 53-72. doi: 10.1002/(SICI) 1096-9861(19980525)395:1<53::AID-CNE5>3. $0 . \mathrm{CO} ; 2-\mathrm{G}$

Kabli, N., Nguyen, T., Balboni, G., O’Dowd, B. F., and George, S. R. (2013). Antidepressant-like and anxiolytic-like effects following activation of the mudelta opioid receptor heteromer in the nucleus accumbens. Mol. Psychiatry doi: 10.1038/mp.2013.115. [Epub ahead of print].

Kim, S. Y., Adhikari, A., Lee, S. Y., Marshel, J. H., Kim, C. K., Mallory, C. S., et al. (2013). Diverging neural pathways assemble a behavioural state from separable features in anxiety. Nature 496, 219-223. doi: 10.1038/nature12018

Kober, H., Barrett, L. F., Joseph, J., Bliss-Moreau, E., Lindquist, K., and Wager, T. D. (2008). Functional grouping and cortical-subcortical interactions in emotion: a meta-analysis of neuroimaging studies. Neuroimage 42, 998-1031. doi: 10. 1016/j.neuroimage.2008.03.059

Lecrubier, Y. (2007). Widespread underrecognition and undertreatment of anxiety and mood disorders: results from 3 European studies. J. Clin. Psychiatry 68(Suppl. 2), 36-41.

LeDoux, J. E. (2000). Emotion circuits in the brain. Annu. Rev. Neurosci. 23, 155184. doi: 10.1146/annurev.neuro.23.1.155

Li, C. I., Maglinao, T. L., and Takahashi, L. K. (2004). Medial amygdala modulation of predator odor-induced unconditioned fear in the rat. Behav. Neurosci. 118, 324-332. doi: 10.1037/0735-7044.118.2.324

Likhtik, E., Stujenske, J. M., Topiwala, M. A., Harris, A. Z., and Gordon, J. A. (2014). Prefrontal entrainment of amygdala activity signals safety in learned fear and innate anxiety. Nat. Neurosci. 17, 106-113. doi: 10.1038/nn.3582

Machado-de-Sousa, J. P., Osorio Fde, L., Jackowski, A. P., Bressan, R. A., Chagas, M. H., Torro-Alves, N., et al. (2014). Increased amygdalar and hippocampal volumes in young adults with social anxiety. PLoS One 9:e88523. doi: 10. 1371/journal.pone.0088523

McDonald, A. J. (1983). Neurons of the bed nucleus of the stria terminalis: a golgi study in the rat. Brain Res. Bull. 10, 111-120. doi: 10.1016/0361-9230(83) 90082-5

McNaughton, N., and Gray, J. A. (2000). Anxiolytic action on the behavioural inhibition system implies multiple types of arousal contribute to anxiety. $J$. Affect. Disord. 61, 161-176. doi: 10.1016/s0165-0327(00)00344-x

Menard, J., and Treit, D. (1996). Lateral and medial septal lesions reduce anxiety in the plus-maze and probe-burying tests. Physiol. Behav. 60, 845-853. doi: 10. 1016/0031-9384(96)00138-2

Moller, C., Wiklund, L., Sommer, W., Thorsell, A., and Heilig, M. (1997). Decreased experimental anxiety and voluntary ethanol consumption in rats following central but not basolateral amygdala lesions. Brain Res. 760, 94-101. doi: 10 . 1016/s0006-8993(97)00308-9

Moreira, C. M., Masson, S., Carvalho, M. C., and Brandao, M. L. (2007). Exploratory behaviour of rats in the elevated plus-maze is differentially sensitive to inactivation of the basolateral and central amygdaloid nuclei. Brain Res. Bull. 71, 466-474. doi: 10.1016/j.brainresbull.2006.10.004

O’Leary, T. A., Rohsenow, D. J., Martin, R., Colby, S. M., Eaton, C. A., and Monti, P. M. (2000). The relationship between anxiety levels and outcome of cocaine abuse treatment. Am. J. Drug Alcohol Abuse 26, 179-194. doi: 10.1081/ada100100599
Papez, J. W. (1995). A proposed mechanism of emotion. 1937. J. Neuropsychiatry Clin. Neurosci. 7, 103-112.

Pellow, S., Chopin, P., File, S. E., and Briley, M. (1985). Validation of open: closed arm entries in an elevated plus-maze as a measure of anxiety in the rat. $J$. Neurosci. Methods 14, 149-167. doi: 10.1016/0165-0270(85)90031-7

Pitkanen, A. (2000). "Connectivity of the rat amygdaloid complex," in The Amygdala: A Functional Analysis, ed J. P. Aggleton (Oxford: Oxford Univeristy Press), 31-116.

Pobbe, R. L., Defensor, E. B., Pearson, B. L., Bolivar, V. J., Blanchard, D. C., and Blanchard, R. J. (2011). General and social anxiety in the BTBR T+ tf/J mouse strain. Behav. Brain Res. 216, 446-451. doi: 10.1016/j.bbr.2010. 08.039

Price, J. L., and Amaral, D. G. (1981). An autoradiographic study of the projections of the central nucleus of the monkey amygdala. J. Neurosci. 1, 1242-1259.

Privou, C., Knoche, A., Hasenohrl, R. U., and Huston, J. P. (1998). The H1- and H2-histamine blockers chlorpheniramine and ranitidine applied to the nucleus basalis magnocellularis region modulate anxiety and reinforcement related processes. Neuropharmacology 37, 1019-1032. doi: 10.1016/s0028-3908(98)00 $087-2$

Qin, S., Young, C. B., Duan, X., Chen, T., Supekar, K., and Menon, V. (2013). Amygdala subregional structure and intrinsic functional connectivity predicts individual differences in anxiety during early childhood. Biol. Psychiatry doi: 10. 1016/j.biopsych.2013.10.006. [Epub ahead of print].

Roberts, G. W., Woodhams, P. L., Polak, J. M., and Crow, T. J. (1982). Distribution of neuropeptides in the limbic system of the rat: the amygdaloid complex. Neuroscience 7, 99-131. doi: 10.1016/0306-4522(82)90156-7

Satpute, A. B., Mumford, J. A., Naliboff, B. D., and Poldrack, R. A. (2012). Human anterior and posterior hippocampus respond distinctly to state and trait anxiety. Emotion 12, 58-68. doi: 10.1037/a0026517

Schmitt, U., and Hiemke, C. (1998). Combination of open field and elevated plusmaze: a suitable test battery to assess strain as well as treatment differences in rat behavior. Prog. Neuropsychopharmacol. Biol. Psychiatry 22, 1197-1215. doi: 10. 1016/s0278-5846(98)00051-7

Schoenfeld, T. J., Hsueh, B., Runkle, M. B., Kloth, A. D., Wang, S. S., and Gould, E. (2013). Gap junctions in the ventral hippocampus-medial prefrontal cortex pathway are involved in anxiety regulation. SfN Abstr. 2013

Seidenbecher, T., Laxmi, T. R., Stork, O., and Pape, H. C. (2003). Amygdalar and hippocampal theta rhythm synchronization during fear memory retrieval. Science 301, 846-850. doi: 10.1126/science.1085818

Senn, V., Wolff, S. B. E., Herry, C., Grenier, F., Ehrlich, I., Grundemann, J., et al. (2014). Long-range connectivity defines behavioral specificity of amygdala neurons. Neuron 81, 428-437. doi: 10.1016/j.neuron.2013.11.006

Silveira, M. C., Sandner, G., and Graeff, F. G. (1993). Induction of Fos immunoreactivity in the brain by exposure to the elevated plus-maze. Behav. Brain Res. 56, 115-118. doi: 10.1016/0166-4328(93)90028-O

Smythe, J. W., Colom, L. V., and Bland, B. H. (1992). The extrinsic modulation of hippocampal theta depends on the coactivation of cholinergic and GABA-ergic medial septal inputs. Neurosci. Biobehav. Rev. 16, 289-308. doi: 10.1016/s01497634(05)80203-9

Somerville, L. H., Whalen, P. J., and Kelley, W. M. (2010). Human bed nucleus of the stria terminalis indexes hypervigilant threat monitoring. Biol. Psychiatry 68, 416-424. doi: 10.1016/j.biopsych.2010.04.002

Sylvers, P., Lilienfeld, S. O., and Laprairie, J. L. (2011). Differences between trait fear and trait anxiety: implications for psychopathology. Clin. Psychol. Rev. 31, 122-137. doi: 10.1016/j.cpr.2010.08.004

Tang, J., Ma, Y., Guo, Y., Ahmed, N. I., Yu, Y., and Wang, J. (2013). Association of aggression and non-suicidal self injury: a school-based sample of adolescents. PLoS One 8:e78149. doi: 10.1371/journal.pone.0078149

Treit, D., Aujla, H., and Menard, J. (1998). Does the bed nucleus of the stria terminalis mediate fear behaviors? Behav. Neurosci. 112, 379-386. doi: 10. 1037/0735-7044.112.2.379

Tye, K. M., Prakash, R., Kim, S. Y., Fenno, L. E., Grosenick, L., Zarabi, H., et al. (2011). Amygdala circuitry mediating reversible and bidirectional control of anxiety. Nature 471, 358-362. doi: 10.1038/nature09820

van Dijk, A., Klanker, M., van Oorschot, N., Post, R., Hamelink, R., Feenstra, M. G., et al. (2013). Deep brain stimulation affects conditioned and unconditioned anxiety in different brain areas. Transl. Psychiatry 3:e289. doi: 10.1038/tp. 2013.56 
Walker, D. L., and Davis, M. (1997a). Anxiogenic effects of high illumination levels assessed with the acoustic startle response in rats. Biol. Psychiatry 42, 461-471. doi: 10.1016/s0006-3223(96)00441-6

Walker, D. L., and Davis, M. (1997b). Double dissociation between the involvement of the bed nucleus of the stria terminalis and the central nucleus of the amygdala in startle increases produced by conditioned versus unconditioned fear. $J$. Neurosci. 17, 9375-9383.

Walter, A., Mai, J. K., Lanta, L., and Gorcs, T. (1991). Differential distribution of immunohistochemical markers in the bed nucleus of the stria terminalis in the human brain. J. Chem. Neuroanat. 4, 281-298. doi: 10.1016/08910618(91)90019-9

Woodhams, P. L., Roberts, G. W., Polak, J. M., and Crow, T. J. (1983). Distribution of neuropeptides in the limbic system of the rat: the bed nucleus of the stria terminalis, septum and preoptic area. Neuroscience 8, 677-703. doi: 10. 1016/0306-4522(83)90003-9

Yassa, M. A., Hazlett, R. L., Stark, C. E., and Hoehn-Saric, R. (2012). Functional MRI of the amygdala and bed nucleus of the stria terminalis during conditions of uncertainty in generalized anxiety disorder. J. Psychiatr. Res. 46, 1045-1052. doi: 10.1016/j.jpsychires.2012.04.013

Conflict of interest statement: The author declares that the research was conducted in the absence of any commercial or financial relationships that could be construed as a potential conflict of interest.

Received: 27 January 2014; accepted: 15 March 2014; published online: 01 April 2014 Citation: Adhikari A (2014) Distributed circuits underlying anxiety. Front. Behav. Neurosci. 8:112. doi: 10.3389/fnbeh.2014.00112

This article was submitted to the journal Frontiers in Behavioral Neuroscience.

Copyright ( $) 2014$ Adhikari. This is an open-access article distributed under the terms of the Creative Commons Attribution License (CC BY). The use, distribution or reproduction in other forums is permitted, provided the original author(s) or licensor are credited and that the original publication in this journal is cited, in accordance with accepted academic practice. No use, distribution or reproduction is permitted which does not comply with these terms. 